\title{
Use of Parathyroid Hormone and Rehabilitation Reduces Subsequent Vertebral Body Fractures after Balloon Kyphoplasty
}

\author{
Masaki Ueno ${ }^{1}$, Emi Toriumi ${ }^{2}$, Aki Yoshii ${ }^{2}$, Yuki Tabata ${ }^{2}$, Takeshi Furudate ${ }^{1}$, Yusuke Tajima ${ }^{1}$ \\ ${ }^{1}$ Department of Orthopaedic Surgery, Machida Keisen Hospital, Machida, Japan \\ ${ }^{2}$ Department of Rehabilitation, Machida Keisen Hospital, Machida, Japan
}

Study Design: Retrospective cohort study.

Purpose: To evaluate the efficacy of our current prophylactic strategy by investigating the incidence of subsequent vertebral body fractures (SVBFs) following balloon kyphoplasty (BKP).

Overview of Literature: Although extensive studies have investigated the risk factors for SVBFs after BKP, few have reported on postoperative therapies to prevent SVBFs and have evaluated their effectiveness.

Methods: This study enrolled 273 patients who underwent an initial BKP. To treat osteoporosis, parathyroid hormone (PTH) administration was started 1-2 weeks before BKP and continued for at least 6 months postoperatively. Corsets were applied for 3 months after the procedure. Rehabilitative interventions, including hip range-of-motion training, muscle strengthening exercises, and motion/ posture instruction, were started from the preoperative assessment time point and resumed 3 hours postoperatively. Corsets were used in all patients. Therefore, no grouping based on corset use was performed. PTH was used in 180 patients, and they were divided into the following two groups: PTH user group and PTH nonuser group. Rehabilitative interventions were provided to all patients for a median duration of 17 days. Patients who underwent rehabilitative intervention for $<17$ and $\geq 17$ days were included in the short-term and long-term intervention groups, respectively. The incidences of SVBFs for these four groups were compared.

Results: SVBF occurred in 29 patients (10.6\%). The SVBF incidence among patients who were prescribed all three prophylactic measures was $6.2 \%$. The PTH user group had a significantly lower incidence of distant vertebral body fractures as compared to the PTH nonuser group. The long-term rehabilitation group had a significantly lower incidence of SVBFs and adjacent vertebral body fractures within 50 postoperative days than the short-term group.

Conclusions: A 17-day or longer rehabilitative intervention may lower the risk of early adjacent vertebral body fractures, and the use of PTH may reduce the risk of distant vertebral body fractures.

Keywords: Balloon vertebroplasty; Compression fracture; Rehabilitation; Parathyroid hormone; Teriparatide

Received Nov 30, 2020; Revised Jan 8, 2021; Accepted Jan 29, 2021

Corresponding author: Masaki Ueno

Department of Orthopaedic Surgery, Machida Keisen Hospital, 2-1-47 Minamimachida, Machida, Tokyo 194-0005, Japan

Tel: +81-42-795-1668, Fax: +81-42-796-2680, E-mail: ueno@machida-keisen.com 


\section{Introduction}

Balloon kyphoplasty (BKP) for osteoporotic vertebral compression fractures has been demonstrated to effectively reduce pain right from the early postoperative period, but it is commonly recognized that the technique is commonly associated with a postoperative complication of subsequent vertebral body fractures (SVBFs) [1-6]. Although it remains unclear whether new fractures are attributable to BKP or to the natural progression of osteoporosis, it is true that new vertebral fractures can occur during the follow-up period after BKP. The use of parathyroid hormone (PTH) and corset wearing have been reported as prophylactic measures for SVBFs following BKP and have been evaluated for their preventive efficacy. However, no single measure has demonstrated an apparent preventive effect. Since the introduction of BKP, we have considered it challenging to prevent SVBFs with a single prophylactic measure and have employed a combination of PTH, corset wearing, and rehabilitative interventions as a basic strategy. Thus, this study aimed to evaluate the efficacy of the preventive strategy by investigating and performing an exploratory analysis of the postoperative outcome of BKP at Machida Keisen Hospital.

\section{Materials and Methods}

\section{Patients}

Among the 876 patients diagnosed with fresh vertebral compression fracture at Machida Keisen Hospital from April 2014 to September 2018, 476 underwent operative treatment. Of these, 292 underwent initial BKP for the vertebral compression fractures caused by primary osteoporosis; of these, 273 (82 men and 191 women; mean age at surgery, 81.4 years; range, 66-101 years) who were followed up for at least 1 year were included in this study. The affected vertebrae were in the mid- to lower thoracic spine (T4-10) in 22 patients $(8.1 \%)$, the thoracolumbar junction (T11-L2) in 186 patients (68.1\%), and the lower lumbar spine (L3-5) in 65 patients (23.8\%). The mean preoperative bone mineral density (BMD) relative to the young adult mean (YAM) was $76.9 \%(33 \%-141 \%)$ in the lumbar spine and $67.8 \%(31 \%-136 \%)$ in the femur. The number of existing fractured vertebrae at the time of injury was one in 21 patients, two in 10 patients, and $\geq 3$ in eight patients. The average duration of conservative therapy from the time of presentation to our hospital was 24.1 days (range, 15-386 days), with 245 patients (89.7\%) having a definite date of injury. The mean time from injury to surgery was 31.6 days (range, 22-355 days). The mean number of hospitalization days was 35.8 days (range, 2-241 days).

\section{Prophylactic measures}

We recommended that all patients be treated with PTH (teriparatide) for osteoporosis and be given weekly or daily doses as per their preferences irrespective of whether they underwent BKP. PTH therapy was started 1-2 weeks before BKP and continued until at least 6 months after the surgery. Those who refused PTH continued to undergo the existing antiosteoporosis treatment if any was being administered at the time of injury, and they were prescribed once-weekly alendronate if no other treatment was administered. After surgery, Jewett-type corsets were applied to the thoracolumbar junction or hard corsets were applied to other parts for 3 months. Patients were instructed to always wear the corset, except when bathing, and were taught how to wear the corset while in the supine position. Rehabilitative interventions, including hip range-of-motion training, muscle strengthening exercises, and movement/posture instructions, were undertaken from the time of preoperative assessment and resumed 3 hours after the surgery (Table 1 and Figs. 1, 2). The patients were allowed to decide how much rest he/she should have based on the degree of pain, except for some restrictions, such as not lifting heavy objects. The periods of hospitalization and rehabilitation were determined at the patient's request. Rehabilitation was performed every day during the hospitalization period. For patients transferred to outpatient care (including the outpatient rehabilitation program at our hospital), the number of days of outpatient visits was counted as the number of days of rehabilitation.

\section{Endpoint}

SVBFs were defined as all morphological fractures of vertebral bodies, including adjacent and distant vertebral bodies, occurring during the postoperative follow-up period, irrespective of the presence of clinical symptoms or mechanism of injury, accompanied by patient complaints of low back pain or radiographic reduction of the verte- 
Table 1. Details of the rehabilitative interventions ( 2 units; 40 minutes, in total)

\begin{tabular}{|c|c|c|}
\hline Item & Duration (min) & Details \\
\hline Assessment & 5 & Posture, muscle strength, and gait ability assessments \\
\hline Hip range-of-motion training & 5 & - \\
\hline Muscle-strengthening training & 10 & See Fig. 1 \\
\hline Basic movement training & 20 & Movement instruction (see Fig. 2), posture instruction, and gait training \\
\hline
\end{tabular}

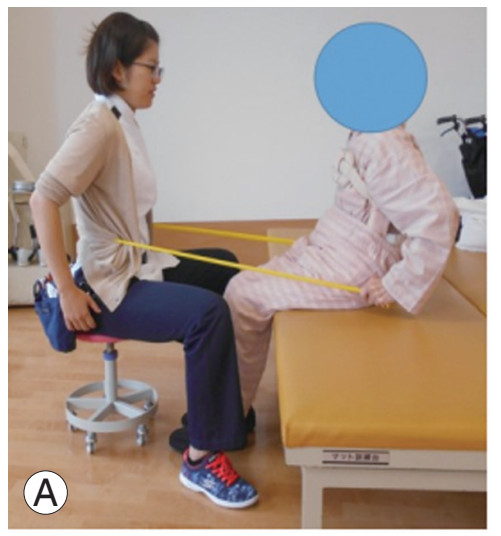

Fig. 1. (A, B) Training to strengthen abdominal and back muscle groups by shoulder joint extension/flexion exercise using TheraBand.

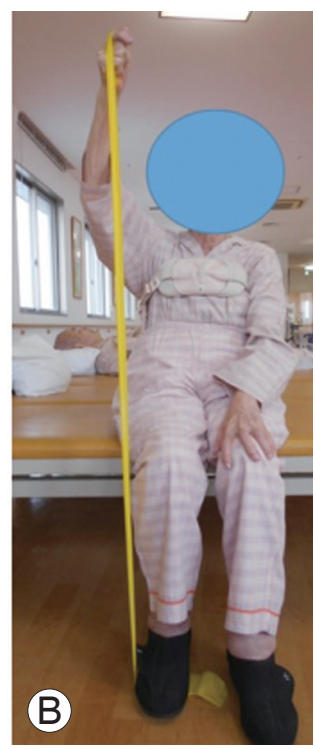

bral height at follow-up visits ( 1 week; 1,3 , and 6 months; and 1 year postoperatively) and determined to be a fresh fracture on magnetic resonance imaging.

\section{Statistics}

Corsets were used in all patients. Therefore, no grouping based on corset use was performed. Rehabilitative interventions were performed for a median duration of 17 days, and patients who received the interventions for $<17$ days and those who received the intervention for $\geq 17$ days were categorized into the short- and long-term intervention groups, respectively. Basic patient data, including age and $\mathrm{BMD} / \mathrm{YAM}$, and the incidence of SVBFs were compared among the following four groups: the no-PTH/ short-term intervention (S), no-PTH/long-term intervention (L), PTH/short-term intervention (PS), and PTH/ long-term intervention (PL) groups. The distribution of the time of onset of SVBFs, divided into adjacent and distant vertebral body fractures, was compared among these four groups.

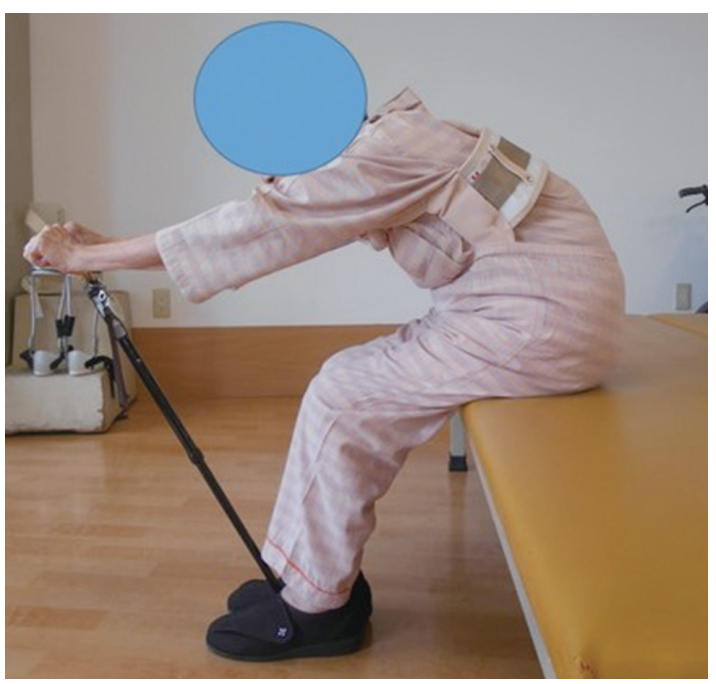

Fig. 2. Movement instruction to promote anterior pelvic tilt and hip flexion.

Statistical analyses were performed using IBM SPSS Statistics ver. 20.0 (IBM Japan Business Services Co. Ltd., Tokyo, Japan). For comparisons among the four groups, one-way analysis of variance, followed by multiple comparison tests using the Tukey or Games-Howell method, was performed for age, BMD (\%YAM), number of existing fractured vertebrae, and duration of conservative treatment. A chi-square test was performed by analyzing variables such as sex, level of injured vertebrae, and number of SVBFs.

\section{Ethics statements}

The protocol of this study was reviewed and approved by the Institutional Review Board of Machida Keisen Hospital (IRB approval no., 2019-001), and informed consent was waived because of the retrospective study design.

\section{Results}

PTH was used in 180 patients (weekly in 132 and daily in 
Table 2. Basic data of the four groups

\begin{tabular}{|c|c|c|c|c|c|}
\hline Characteristic & $S$ group $(n=48)$ & $L$ group $(n=45)$ & PS group ( $n=83$ ) & $P L$ group $(n=97)$ & $p$-value \\
\hline Age (yr) & $77.9 \pm 8.3$ & $82.3 \pm 9.4$ & $79.8 \pm 6.6$ & $84.0 \pm 6.6$ & 0.00 \\
\hline Sex & & & & & 0.11 \\
\hline Female & 28 & 32 & 65 & 66 & \\
\hline Male & 20 & 13 & 18 & 31 & \\
\hline \multicolumn{6}{|l|}{ Level of injured vertebrae } \\
\hline $\mathrm{T} 4-10$ & 2 & 2 & 12 & 6 & \\
\hline T10-L2 & 33 & 26 & 59 & 68 & 0.03 \\
\hline L3-5 & 13 & 17 & 12 & 23 & \\
\hline \multicolumn{6}{|l|}{ BMD/YAM (\%) } \\
\hline Lumber spine & $83.3 \pm 25.4$ & $82.2 \pm 21.5$ & $68.7 \pm 18.9$ & $73.3 \pm 17.2$ & 0.00 \\
\hline Femur & $72.6 \pm 14.7$ & $67.7 \pm 20.2$ & $66.7 \pm 14.4$ & $64.2 \pm 10.2$ & 0.04 \\
\hline No. of existing fractured vertebrae & $2.3 \pm 0.9$ & $2.5 \pm 1.1$ & $2.3 \pm 0.9$ & $2.3 \pm 0.8$ & 0.60 \\
\hline Duration of conservative therapy (day) & $19.4 \pm 34.8$ & $20.0 \pm 55.3$ & $16.3 \pm 17.7$ & $40.5 \pm 76.3$ & 0.21 \\
\hline No. of SVBFs & 7 & 6 & 10 & 6 & 0.34 \\
\hline Adjacent vertebrae & 5 & 1 & 7 & 6 & 0.43 \\
\hline Distant vertebrae & 2 & 5 & 3 & 0 & 0.00 \\
\hline
\end{tabular}

Values are presented as mean \pm standard deviation or number. A $p$-value $<0.05$ is statistically significant.

PTH, parathyroid hormone; S group, no-PTH/short-term intervention group; L group, no-PTH/long-term intervention group: PS group, PTH/short-term intervention group; PL group, PTH/short-term intervention group; BMD/YAM, bone mineral density/young adult mean; SVBF, subsequent vertebral body fracture.

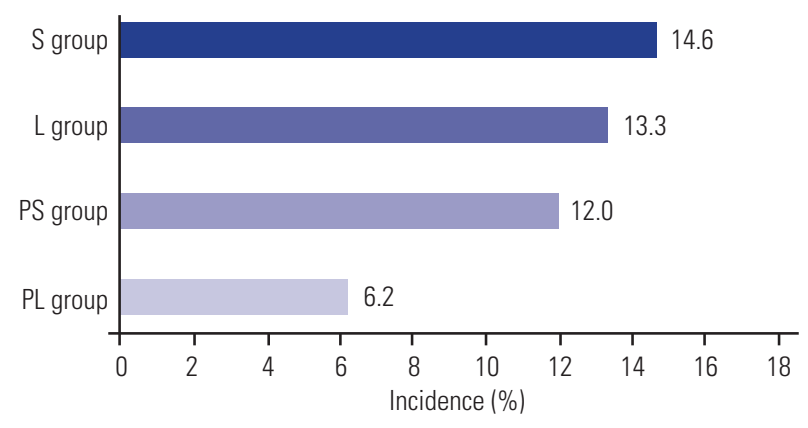

Fig. 3. Incidence of subsequent vertebral body fractures in the four groups. The PL group had the lowest incidence, which was less than half that of the $S$ group. PTH, parathyroid hormone; S group, no-PTH group with short-term rehabilitation; L group, no-PTH group with long-term rehabilitation; PS group, PTH group with short-term rehabilitation; PL group, PTH group with long-term rehabilitation.

48). All patients were prescribed corsets, including hard ones, such as the Jewett-type corset $(\mathrm{n}=263)$, and soft ones $(\mathrm{n}=10)$ that were used because they had already been prepared by the previous physician or because of strong patient preference. Rehabilitative interventions were performed in all patients for an average duration of 31.1 days (range, 1-238 days). The basic data of the four groups are shown in Table 2. The mean ages of the long-term rehabilitation groups (L and PL) were significantly higher than those of the short-term rehabilitation groups (S and PS groups). BMD/YAM was significantly lower in the PTH groups (PS and PL groups) than in the no-PTH groups (S and $\mathrm{L}$ groups).

SVBFs occurred in 29 of 273 patients (10.6\%), including seven of 48 patients (14.6\%) in the S group, six of 45 patients (13.3\%) in the L group, 10 of 83 patients (12.0\%) in the PS group, and six of 97 patients (6.2\%) in the PL group. Thus, the PL group had the lowest incidence of SVBFs (Fig. 3).

Of the 29 SVBFs, 19 (65.5\%) were adjacent vertebral body fractures, and the remaining 10 (34.5\%) were distant vertebral body fractures. The mean postoperative time to onset was 72.7 days for all SVBFs, 57.6 days for adjacent vertebral body fractures, and 101.2 days for distant vertebral body fractures. The distribution of the time to onset of SVBFs showed that $>50 \%$ of all SVBFs occurred within 50 days of surgery, of which $>81 \%$ were adjacent vertebral body fractures (Fig. 4). When SVBFs were divided into adjacent and distant vertebral body fractures and their incidences were compared between the short- and long-term rehabilitation groups, the long-term groups had significantly lower incidences of all SVBFs and adjacent vertebral body fractures within 50 days of surgery than the short-term groups (Fig. 


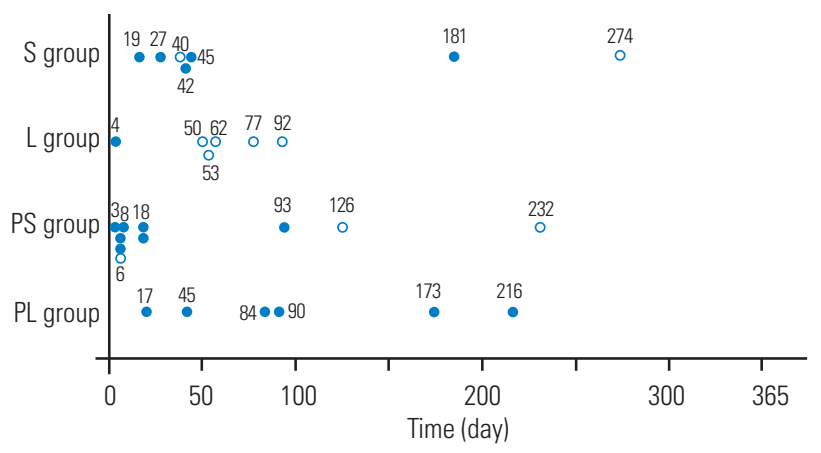

Fig. 4. Distribution of the time of onset of subsequent vertebral body fractures in the four groups. The blue dots indicate adjacent vertebral body fractures, and the white dots indicate distant vertebral body fractures. The postoperative onset days of fractures were plotted along with the numbers of vertebral body fractures. The occurrence of adjacent vertebral body fractures in the early postoperative period was less frequent in the $L$ and $P L$ groups than $S$ and PS groups, in which rehabilitation was performed for $\geq 17$ days. Few distant vertebral body fractures occurred in the PS and PL groups, in which parathyroid hormone (PTH) was used. S group, no-PTH group with short-term rehabilitation; L group, no-PTH group with long-term rehabilitation; PS group, PTH group with short-term rehabilitation; PL group, PTH group with long-term rehabilitation.

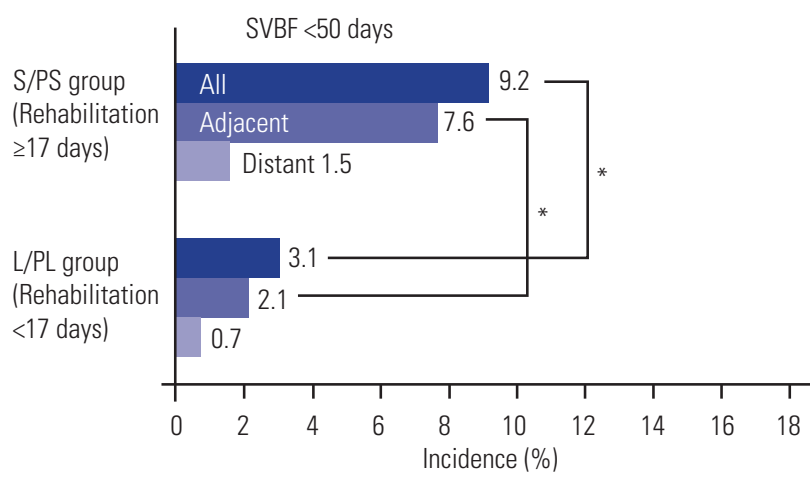

Fig. 5. Incidence of subsequent vertebral body fractures within 50 postoperative days in the long- and short-term rehabilitation groups. The long-term groups had a significantly lower incidence of adjacent vertebral body fractures (and all SVBFs) than the short-term groups. SVBF, subsequent vertebral body fractures; PTH, parathyroid hormone; S group, no-PTH group with short-term rehabilitation; L group, no-PTH group with long-term rehabilitation; PS group, PTH group with short-term rehabilitation; PL group, PTH group with long-term rehabilitation. ${ }^{*} p<0.05$.

5). When the incidences were compared between those who did and did not use PTH, the PTH group had a significantly lower incidence of distant vertebral body fractures as compared to the no-PTH group (Fig. 6).

\section{Discussion}

The present study showed that the incidence of SVBFs after BKP at our hospital was $10.6 \%$, the lowest among all Japanese reports of the same size that could be identified

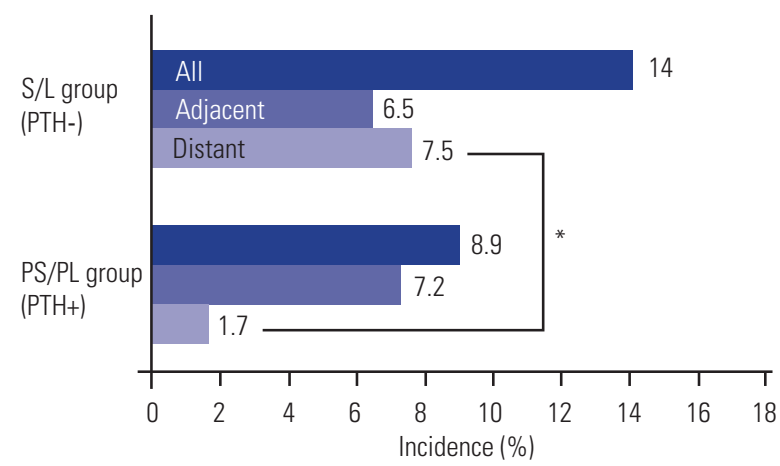

Fig. 6. Incidence of subsequent vertebral body fractures in the parathyroid hormone (PTH) and no-PTH groups. The PTH groups had a significantly lower incidence of distant vertebral body fractures than the no-PTH groups. S group, no-PTH group with short-term rehabilitation; L group, no-PTH group with longterm rehabilitation; PS group, PTH group with short-term rehabilitation; PL group, PTH group with long-term rehabilitation. ${ }^{*} p<0.05$.

in our literature search. The incidence was even lower $(6.2 \%)$ in patients who were prescribed all three prophylactic measures $(\mathrm{PTH}$, corset wearing, and rehabilitative interventions) and was less than half of that in patients with corset wearing alone (no-PTH, short-term rehabilitation). Although most SVBFs occurred within 100 days of surgery, the long-term rehabilitation groups were less likely to experience adjacent vertebral body fractures within 50 days of surgery than the short-term groups. Moreover, patients who used PTH had an incidence of adjacent vertebral body fractures similar to that seen in those who did not use PTH but were less likely to experience distant vertebral body fractures during the study period. On the basis of the currently available data, it is unlikely that PTH was effective enough to prevent adjacent vertebral body fractures at the average time of their onset. The reduced incidence of adjacent vertebral body fractures in the early postoperative period is more likely to have been caused by the rehabilitation. Simultaneously, the average duration of rehabilitation was about 30 days, and it is unlikely that its preventive effect on fractures would last so long thereafter. Thus, it is likely that the reduced incidence of distant vertebral body fractures was attributable to PTH. The combined preventive effect of PTH and rehabilitation may be attributable to the synergy of these two measures. In particular, although neither PTH nor rehabilitation appeared effective in preventing all SVBFs throughout the study period, it can be speculated that a 17-day or longer rehabilitation program reduced early adjacent vertebral body fractures, and PTH use reduced distant vertebral body fractures during the study period, resulting in a low 
overall SVBF incidence of about 6\%.

Although extensive reports have been published on the risk factors for SVBFs after BKP, few have reported on postoperative therapies to prevent SVBFs and evaluated their effectiveness. Although some reports suggested the efficacy of the prophylactic use of PTH [7-10], negative results have also been reported $[11,12]$. Corsets are commonly used as standard postoperative therapy to prevent the worsening of kyphotic deformities by limiting forward bending of the trunk during posture correction [13]. However, no single measure has demonstrated a clear preventive effect on SVBFs. Moreover, no consensus has been reached on any single risk factor for SVBFs, and it is speculated that a wide variety of factors, in addition to the reported risk factors, such as preoperative local kyphosis, vertebral height restoration rate, $\mathrm{BMD}$, and spinal alignment at the time of injury [14-16], are involved in the occurrence of SVBFs in the actual clinical setting. However, it is impossible to intervene in preoperative risk factors, except to avoid BKP in patients judged to have these risk factors. If postoperative prophylactic measures are to be devised, it is important to ignore the nonmodifiable preoperative factors, but a combination of prophylactic measures that target modifiable postoperative factors is expected to be effective.

Although rehabilitation as a prophylactic measure for SVBFs after BKP has not been reported so far, exercise therapy has long and widely been used for preventing osteoporotic compression fractures and is reportedly effective in preventing compression fractures by strengthening the trunk extensor muscles, improving sagittal spinal balance, and preventing the progression of vertebral collapse [17-19]. In patients who have developed compression fractures, kyphosis due to vertebral deformity decreases muscle contractility in the lower back and increases the load on the anterior component of the vertebrae, that is, vertebral bodies, due to the shift of the load line, making subsequent fractures more likely. Therefore, these patients require muscle strengthening training in the lower back and postural instruction. Moreover, standing up from a seated position that involves forward bending of the trunk and that increases pressure stress on the vertebral bodies is considered another mechanism of occurrence of SVBFs [20], implying the need for hip range-of-motion training and movement instruction. The rehabilitation program provided at our hospital as a prophylactic measure for SVBFs after BKP is based on the same concept, and re- habilitative interventions may help in avoiding postures and movements that can cause SVBFs until such time that PTH therapy becomes effective and prevents kyphosis progression via muscle strengthening.

Among the suggested mechanisms of occurrence of SVBFs after BKP, the hypothesis that cemented vertebrae increase the mechanical stress on the adjacent vertebrae $[21,22]$ and the one that SVBFs are attributable to low BMD and kyphosis seem to be consistent with the risk factors recently reported in Japan [23]. The intervention for these factors requires rapid correction of osteoporosis and maintenance of alignment. For rapid correction of osteoporosis, PTH was among the most potent and rapidacting treatments for osteoporosis available at the time of writing this report, with weekly PTH administration being the most effective in preventing new vertebral body fractures [24]. It is widely known that exercise therapy performed as a rehabilitative intervention can increase BMD [25], implying the importance of synergy between exercise and PTH therapies. For the maintenance of spinal alignment, one can expect that the vertebral height can be restored during surgery, but some reports suggest that BKP does not provide sustained alignment maintenance [26], and others report that a high vertebral height restoration rate is a strong risk factor [14]. Nevertheless, postural correction via corset wearing and rehabilitation is the only feasible postoperative intervention. Therefore, we combined the following three prophylactic measures to prevent SVBFs after BKP: PTH use, corset wearing, and rehabilitative interventions. In the present study, the combination of all three prophylactic measures reduced the incidence of SVBFs to as low as around 6\%, suggesting that our combination of prophylactic measures was more successful than those reported previously.

The present study was only an exploratory analysis of the postoperative outcome of BKP performed at our hospital, and the number of fracture events was insufficient for a multivariate analysis. The rehabilitative interventions performed were not completely standardized for the study and might have slightly varied among physical therapists. Further studies are needed to develop a standardized rehabilitation program and identify methods to evaluate its effectiveness.

\section{Conclusions}

The prevalence of SVBFs after BKP in our hospital was 
$10.3 \%$, lower than that previously reported in Japan, and the prevalence was even lower $(6.2 \%)$ in patients prescribed a combined prophylactic measure of PTH use, corset wearing, and rehabilitative interventions. A combination of multiple measures should be considered in the prevention of SVBFs after BKP.

\section{Conflict of Interest}

No potential conflict of interest relevant to this article was reported.

\section{Author Contributions}

MU participated in conception and design, analysis of data, and critical revision of the study. EF, MU, AY, YTabata and YTajima contributed to acquisition of data. MU and TF were surgeons in this study. EF, MU, and AY participated in drafting of the manuscript.

\section{References}

1. Voggenreiter G. Balloon kyphoplasty is effective in deformity correction of osteoporotic vertebral compression fractures. Spine (Phila Pa 1976) 2005;30:2806-12.

2. Bouza C, Lopez T, Magro A, Navalpotro L, Amate JM. Efficacy and safety of balloon kyphoplasty in the treatment of vertebral compression fractures: a systematic review. Eur Spine J 2006;15:1050-67.

3. Garfin SR, Buckley RA, Ledlie J; Balloon Kyphoplasty Outcomes Group. Balloon kyphoplasty for symptomatic vertebral body compression fractures results in rapid, significant, and sustained improvements in back pain, function, and quality of life for elderly patients. Spine (Phila Pa 1976) 2006;31:2213-20.

4. Taylor RS, Fritzell P, Taylor RJ. Balloon kyphoplasty in the management of vertebral compression fractures: an updated systematic review and meta-analysis. Eur Spine J 2007;16:1085-100.

5. Pflugmacher R, Schroeder RJ, Klostermann CK. Incidence of adjacent vertebral fractures in patients treated with balloon kyphoplasty: two years' prospective follow-up. Acta Radiol 2006;47:830-40.

6. Farrokhi MR, Alibai E, Maghami Z. Randomized controlled trial of percutaneous vertebroplasty versus optimal medical management for the relief of pain and disability in acute osteoporotic vertebral compression fractures. J Neurosurg Spine 2011;14:561-9.

7. Ueno M, Nakazawa T, Saito W, et al. Efficacy of weekly teriparatide for secondary vertebral compression fracture following balloon kyphoplasty. J Spine Res 2013;4:1399-404.

8. Yasuoka H, Asazuma T, Imabayashi H, et al. Effect of daily teriparatide dosage following BKP (balloon kyphoplasty) method: can it prevent new vertebral body fractures after BKP? JJASCoL 2014;27:116-7.

9. Sano S, Ichimura S, Hasegawa M, et al. The evaluation of new vertebral fracture after balloon kyphoplasty. J Spine Res 2015;6:1076-82.

10. Kong M, Zhou C, Zhu K, et al. 12-Month teriparatide treatment reduces new vertebral compression fractures incidence and back pain and improves quality of life after percutaneous kyphoplasty in osteoporotic women. Clin Interv Aging 2019;14:1693-703.

11. Nakayama Y, Ota H, Matsumoto Y, et al. The surgical outcome of balloon kyphoplasty (BKP): minimum 2 years follow-up. J Spine Res 2016;7:1727-30.

12. Hirose T, Yamashita A, Harada T, et al. Strategies to prevent subsequent vertebral body fractures after balloon kyphoplasty: evaluation of the effect of teriparatide. Orthop Traumatol 2016;65:833-6.

13. Usui M, Takido K, Matsuura T, et al. The effect of the throraco-lumbar orthosis on the mobility of the thoracolumbar junction: Damen corset, Jewett brace and cast. J Jpn Clin Orthop Assoc 2014;39:116-9.

14. Oishi Y, Murase M, Hayashi Y, et al. Risk factors for the early subsequent adjacent vertebral fractures after balloon kyphoplasty. J Spine Res 2013;4:1789-92.

15. Sawada T, Nakano K, Asari T, Kawagishi T. Bone mineral density based study of early vertebral fracture after balloon kyphoplasty. J Spine Res 2017;8:1164-6.

16. Omi H, Nakano K, Nakahara K, et al. Risk factors of early adjacent vertebral fracture after balloon kyphoplasty. J Spine Res 2014;5:930-3.

17. Sinaki M, Itoi E, Wahner HW, et al. Stronger back muscles reduce the incidence of vertebral fractures: a prospective 10 year follow-up of postmenopausal women. Bone 2002;30:836-41.

18. Isomi T, Sasao Y, Miura T, et al. QOL scale and standing sagittal plane alignment of the thoracolumbar kyphosis in patients with osteoporotic vertebral fractures, and the effect of therapeutic exercise. Os- 
teoporo Jpn 2007;15:583-90.

19. Akabane Y, Shukunami T, Shinoda M, et al. Therapeutic exercise for osteoporotic spinal compression fractures: vertebral body compression deformity inhibitory effect. Jpn J Phys Ther 2010;44:527-33.

20. Nachemson AL. The lumbar spine: an orthopaedic challenge. Spine 1976;1:59-71.

21. Wilson DR, Myers ER, Mathis JM, et al. Effect of augmentation on the mechanics of vertebral wedge fractures. Spine (Phila Pa 1976) 2000;25:158-65.

22. Belkoff SM, Mathis JM, Fenton DC, Scribner RM, Reiley ME, Talmadge K. An ex vivo biomechanical evaluation of an inflatable bone tamp used in the treatment of compression fracture. Spine (Phila Pa 1976) 2001;26:151-6.

23. Kayanja MM, Evans K, Milks R, Lieberman IH. Adjacent level load transfer following vertebral augmentation in the cadaveric spine. Spine (Phila Pa 1976) 2006;31:E790-7.
24. Committee for the Development of Guidelines for Prevention and Treatment of Osteoporosis. Teriparatide acetate. In: Committee for the Development of Guidelines for Prevention and Treatment of Osteoporosis, editor. Guidelines for prevention and treatment of osteoporosis. Tokyo: Life Science Publishing; 2015. p.116.

25. Kelley GA, Kelley KS, Kohrt WM. Effects of ground and joint reaction force exercise on lumbar spine and femoral neck bone mineral density in postmenopausal women: a meta-analysis of randomized controlled trials. BMC Musculoskelet Disord 2012;13:177.

26. Friedrich HC, Friedrich HJ, Kneisel P, Drumm J, Pitzen T. Balloon kyphoplasty improves back pain but does not result in a permanent realignment of the thoracolumbar spine. Cent Eur Neurosurg 2011;72:176-80. 